\title{
A CONSISTENT CONSEQUENCE OF AD
}

\author{
BY \\ E. L. BULL AND E. M. KLEINBERG ${ }^{1}$
}

\begin{abstract}
We are concerned with the existence of a normal measure concentrating on the $\omega$-closed unbounded sets. We strengthen the known result that the axiom of choice implies no such measure exists. It is shown that the existence of these measures is consistent (relative to a large cardinal). In particular, $\boldsymbol{x}_{2}$ may admit exactly two normal measures: one which contains the $\omega$-closed unbounded sets and the other, the $\aleph_{1}$-closed unbounded sets. This property of $\boldsymbol{X}_{2}$ is a well-known consequence of $\mathrm{AD}$.
\end{abstract}

A well-known consequence of the axiom of determinacy (AD) is that $\boldsymbol{\kappa}_{2}$ is a measurable cardinal having exactly two normal measures [2]. One of the normal measures is generated by the collection of $\omega$-closed unbounded sets, and the other is generated by the $\kappa_{1}$-closed unbounded sets. One might ask if the consistency of this property follows from a set theoretic assumption which is less suspect than AD.

Of particular interest is the existence of a normal measure which contains all $\omega$-closed unbounded sets. We exclude the degenerate case where the cardinal in question is $\aleph_{1}$. There the $\omega$-closed sets coincide with the closed sets, so that every normal measure on $\aleph_{1}$ contains the $\omega$-closed unbounded filter.

In $\S 1$, we observe that the existence of such a normal measure implies a particular partition relation holds. We then show that the partition is inconsistent with the axiom of choice.

In $\$ 2$ is proved the consistency, relative to a large cardinal, of the existence of an inaccessible cardinal $\kappa$ having exactly two normal measures, one of which contains the $\omega$-closed unbounded sets. The large cardinal required is a measurable cardinal which has a normal measure concentrating on measurables.

The techniques of $\$ 2$ can be modified to obtain a model where the following assertion is true:

" $\aleph_{2}$ has exactly two normal measures, one of which contains

all $\omega$-closed unbounded sets and the other contains the

$\aleph_{1}$-closed unbounded sets."

Received by the editors January 13, 1976 and, in revised form, June 23, 1977.

AMS (MOS) subject classifications (1970). Primary 02K05, 02K35, 04A20.

${ }^{1}$ Research supported by NSF Grants MCS76-5525 and 7610430. 
This is the consequence of $\mathrm{AD}$ we prove consistent. The proof is based on the methods of $\$ 2$ and is outlined in $\$ 3$.

We work in Zermelo-Frankel set theory (ZF) without the axiom of choice. The principle of dependent choice is of length $\alpha$ is abbreviated $\mathrm{DC}_{\alpha}$, and $\mathrm{AC}_{\kappa}$ stands for the assertion, "Every $\kappa$-sequence of nonempty sets has a choice function." We shall use the usual terminology for forcing without explicitly defining it. The approach is similar to that of Schoenfield [5]. If $p$ and $q$ are forcing conditions, $p \geqslant q$ means $p$ is stronger (or contains more information) than $q$. The symbol $\mathbf{G}$ is the canonical term in the forcing language which denotes the generic object. The expression $\operatorname{den}_{G} \tau$ stands for the result of evaluating the term $\tau$ with respect to the generic set $G$.

If $P$ is a partial order and $Q$ is a term, in the language appropriate to $P$ which always denotes a partial order, then $P * Q$ denotes the "composition" of $P$ and $Q$. A condition is a pair $\langle p, q\rangle$ where $p \in P$ and $q$ is a term such that $p \Vdash q \in Q$. We choose terms $q$ from some predetermined set, so that $P * Q$ is not a proper class. The ordering is given by

$$
\langle p, q\rangle \leqslant\left\langle p^{\prime}, q^{\prime}\right\rangle \text { iff } p \leqslant p^{\prime} \text { and } p^{\prime} \Vdash q \leqslant q^{\prime} .
$$

Our definitions are standard. Throughout this paper, $\kappa$ will denote a regular, uncountable cardinal. A measure on $\kappa$ is a $\kappa$-additive nonprincipal ultrafilter. A normal measure is a measure with the property that every regressive function on $\kappa$ is constant on a set in the measure. (A function $f$ : $\kappa \rightarrow \kappa$ is regressive if $f(\alpha)<\alpha$ for all nonzero $\alpha \in \kappa$.) A measure $\mu$ is said to concentrate on a subset $A$ of $\kappa$ if $A \in \mu$. Any normal measure concentrates on the set of limit ordinals below $\kappa$. It is easy to show that $\mathrm{AC}_{\kappa}$ implies that any normal measure on $\kappa$ concentrates on the set of regular cardinals below $\kappa$. This is not true without $\mathrm{AC}_{\boldsymbol{\kappa}^{\prime}}$ however. Using the function which takes an ordinal to its cofinality one sees immediately that a normal measure on $\boldsymbol{k}_{2}$ must contain either $\left\{\alpha<\aleph_{2} \mid \operatorname{cf} \alpha=\omega\right\}$ or else $\left\{\alpha<\aleph_{2} \mid\right.$ cf $\left.\alpha=\aleph_{1}\right\}$.

A subset of $\kappa$ is closed if it contains all its limit points. A set is stationary if it intersects every closed unbounded subset of $\kappa$. A normal measure on $\kappa$ contains every closed unbounded subset, so every element of a normal measure is stationary.

If $A \subseteq \kappa$ and $\alpha<\kappa$ is a regular cardinal, we let $(A)_{\alpha}$ be the set of limit points of increasing $\alpha$-sequences of members of $A$. Thus

$$
(\kappa)_{\alpha}=\{\beta<\kappa \mid \operatorname{cf}(\beta)=\alpha\}
$$

is the set of all ordinals below $\kappa$ with cofinality $\alpha$. The set $A$ is $\alpha$-closed if $(A)_{\alpha} \subseteq A$. Observe that $(A)_{\alpha}$ is always $\alpha$-closed. The collection of $\alpha$-closed unbounded subsets of $\kappa$ generates a nonprincipal filter which will be denoted $\mu_{\alpha}$. Our subset $A \subseteq \kappa$ is $\alpha$-stationary if $A$ intersects every member of $\mu_{\alpha}$.

The next lemma will be useful later. Its proof is routine.

I FMMA $\mathrm{O}$ I of "he a normal measure on $\kappa$. Then $u \subset u$ iff $(\kappa)$. $\in u$. 
1. Suppose $\nu$ is a normal measure on $\kappa$. If the axiom of choice holds, then $\nu$ concentrates on $\{\alpha<\kappa \mid \alpha$ is regular $\}$, and hence $\nu$ cannot contain $\mu_{\omega}$. Theorem 1.1 is a sharpening of this observation.

The notation $\kappa \rightarrow(\omega-\mathrm{Sta})^{2}$ means that for every partition $F$ of the two element subsets of $\kappa$ into two pieces $\left(F:[\kappa]^{2} \rightarrow 2\right)$ there is an $\omega$-stationary set $A \subseteq \kappa$ which is homogeneous $\left(\left|F^{\prime \prime}[A]^{2}\right|=1\right)$. The condition $\kappa \rightarrow(\omega-\mathrm{Sta})^{2}$ is a strengthening of the large cardinal properties $\kappa \rightarrow(\mathrm{Sta})^{2}$ and $\kappa \rightarrow(\kappa)^{2}$. These are well known equivalents of ineffability and weak compactness, respectively. Both ineffable cardinals and weakly compact cardinals are compatible with AC. In fact, they are consistent with $V=L$. These facts contrast strikingly with Theorem 1.1.

A well-known theorem of Rowbottom states that any partition $F:[\kappa]^{2} \rightarrow 2$ has a homogeneous set which is an element of $\nu$. If $\mu_{\omega} \subseteq \nu$, such a set must be $\omega$-stationary. Using the axiom of choice we will construct a partition which has no $\omega$-stationary homogeneous set.

THEOREM 1.1. $\mathrm{AC}_{\kappa}$ implies $\kappa \nrightarrow(\omega-\mathrm{Sta})^{2}$.

Proof. Use $\mathrm{AC}_{\kappa}$ to choose, for each $\alpha<\kappa$ with $\operatorname{cf}(\alpha)=\omega$, an $\omega$-sequence $\left\langle\alpha_{n} \mid n \in \omega\right\rangle$ which is cofinal in $\alpha$. Define $F:[\kappa]^{2} \rightarrow 2$ by $F\left(\{\alpha, \beta\}_{<}\right)=0$ if $\operatorname{cf}(\alpha)=\operatorname{cf}(\beta)=\omega$, and if $m$ is the least integer $i$ such that $\alpha_{i} \neq \beta_{i}$ then $\alpha_{m}>\beta_{m}$. Otherwise, take $F(\{\alpha, \beta\})=1$.

Let $A$ be an $\omega$-stationary subset of $\kappa$. We may assume all elements of $A$ have cofinality $\omega$. Lemmas 1.2 and 1.3 show that $A$ cannot be homogeneous.

LEMMA 1.2. $0 \in F^{\prime \prime}[A]^{2}$.

Proof. For contradiction, assume $0 \notin F^{\prime \prime}[A]^{2}$. Then the function $f_{0}: A \rightarrow \kappa$ given by $f_{0}(\alpha)=\alpha_{0}$ is nondecreasing. Either the range of $f_{0}$ is unbounded, or $f_{0}$ is eventually constant. In the second case, let $\eta_{0}$ be a point beyond which $f_{0}$ is constant and consider $f_{1}:\left(A-\eta_{0}\right) \rightarrow \kappa$ to be defined by $f_{1}(\alpha)=\alpha_{1}$. If $f_{1}$ is eventually constant, find $\eta_{1}$ and proceed to $f_{2}$, etc. Since $\operatorname{cf}(\kappa) \neq \omega$, there must be an integer $n$ such that $f_{n}:\left(A-\eta_{n-1}\right) \rightarrow \kappa$ has unbounded range. Choose the least such $n$. Let $D=\left\{\delta^{\alpha} \mid \alpha \in \kappa\right\}$ be the unbounded set defined as follows:

$\delta^{0}$ is the first element of $A-\eta_{n-1}$, and if $\alpha>0, \delta^{\alpha}$ is the least element of $A-\left(\cup_{\gamma \in \alpha} \delta^{\gamma}\right)$ such that $f_{n}\left(\delta^{\alpha}\right)>\left(\cup_{\gamma \in \alpha} \delta^{\gamma}\right)$.

Our set $A$ is $\omega$-stationary, so $A \cap(D)_{\omega} \neq \varnothing$. Choose $\gamma \in A \cap(D) \omega$. Then $\gamma=\cup_{i \in \omega} \delta^{\alpha(i)}$, where $\delta^{\alpha(i)} \in D$. For some $j \in \omega$,

$$
\gamma_{n}<\delta^{\alpha(j)}<\delta^{\alpha(j+1)}<\gamma
$$

and, by the definition of $D$,

$$
\delta^{\alpha(j)}<f_{n}\left(\delta^{\alpha(j+1)}\right)<\delta^{\alpha(j+1)} .
$$

Consequentlv we have $\delta^{\alpha(j+1)}, \gamma \in A$ and $F\left(\left\{\delta^{\alpha(j+1)}, \gamma\right\}_{-}\right)=0$. 
LeMma 1.3. $1 \in F^{\prime \prime}[A]^{2}$.

Proof. Assume $1 \notin F^{\prime \prime}[A]^{2}$ and define the functions $f_{i}$ as in the previous lemma. The functions now are nonincreasing and must eventually be constant. Take $\eta_{n}$ to be a point beyond which all of the functions $f_{0}, \ldots, f_{n}$ are constant. It follows that $A-\left(\cup_{n \in \omega} \eta_{n}\right)$ can contain at most one point, contradicting the fact that $A$ is unbounded in the regular cardinal $\kappa$.

The preceding proofs used no special property of $\omega$. There is an analogous definition of $\kappa \rightarrow(\alpha-\mathrm{Sta})^{2}$, and the corresponding result is proved just as Theorem 1.1.

THEOREM 1.4. $\mathrm{AC}_{\kappa}$ implies, for all regular $\alpha<\kappa, \kappa \nrightarrow(\alpha-\mathrm{Sta})^{2}$.

2. The goal of this section is to find a model in which there is a normal measure containing $\mu_{\omega}$. This result is stated as Theorem 2.3 . We shall rely heavily on Magidor's notion [1] of iterated Prikry forcing, an extension of Prikry's original method [4]. Let us begin with a discussion of this type of forcing in a fairly general context and return to our main topic after the proof of Lemma 2.2.

Suppose $M$ is a model of $\mathrm{ZF}$ is which there is a set $A$ of measurable cardinals, and with each $\alpha \in A$ there is associated a normal measure $\mu_{\alpha}$. The partial ordering of iterated Prikry forcing will be denoted $\mathcal{P}(A)$. It will be the result of applying to $A$ the inductive definition to be stated shortly. Properties (I)-(III) of $P(A)$ are proved simultaneously with the definition of $\mathscr{P}(A)$. In fact, (III) is necessary to keep the induction going.

(I) Forcing with $\mathscr{P}(A)$ preserves cardinals.

(II) Forcing with $\mathcal{P}(A)$ preserves regular cardinals, except elements of $A$. Every element of $A$ has cofinality $\omega$ in the extension.

(III) If $\mu$ is a measure on $\gamma$ and $\gamma \notin A$, then $\gamma$ remains measurable after forcing with $\mathscr{P}(A)$ and there is a term $\tilde{\mu}$ which always denotes a measure on $\gamma$ extending $\mu$. If, in addition, $\mu$ is normal and $A \cap \gamma \notin \mu$, then $\tilde{\mu}$ is normal also. No new measurable cardinals are created.

(IV) If $\gamma<\cap A$, then forcing with $\mathscr{P}(A)$ adds no new subsets of $\gamma$.

(V) There is a canonical term $\tau$ in the language for forcing with $\mathscr{P}(A \cap \gamma)$ such that

$$
\mathscr{P}(A)=\mathscr{P}(A \cap \gamma) * \tau .
$$

The term $\tau$ always denotes the result of applying in the extension by $\mathscr{P}(A \cap \gamma)$ the definition of $\mathscr{P}(-)$ to the set $A-\gamma$. Abusing notation slightly, we shall write

$$
\mathscr{P}(A)=\mathscr{P}(A \cap \gamma) * \mathscr{P}(A-\gamma) .
$$

The point of property $(\mathrm{V})$ is that $\mathscr{P}(A)$ is truly an interation: One may add $\omega$-sequences to the elements of an initial segment of $A$, stop and evaluate terms. and then proceed with the rest of $A$. 
The definition of $\mathscr{P}(A)$ is by induction on the order type of $A$. A condition will be a sequence $\left\langle p_{\alpha}, P_{\alpha}\right\rangle_{\alpha \in A}$ where each $p_{\alpha}$ is a finite increasing sequence of elements of $\alpha$ and $P_{\alpha}$ is a term in the language for forcing with $\mathscr{P}(A \cap \alpha)$ which always denotes a measure one subset of $\alpha$. That is to say, $O_{\Phi(A \cap \alpha)}$ $\Vdash P_{\alpha} \in \tilde{\mu}_{\alpha}$. Here we are using the inductive hypothesis that $\mathcal{P}(A \cap \alpha)$ satisfies (III). We further require that only finitely many of the $p_{\alpha}$ have nonzero length and

$$
O_{\mathscr{P}(A \cap \alpha)} \text { 叔 "every element of } p_{\alpha} \text { is less than } \cap P_{\alpha} \text {." }
$$

The ordering on $\mathscr{P}(A)$ is given by

$$
\left\langle p_{\alpha}, P_{\alpha}\right\rangle_{\alpha \in A} \leqslant\left\langle q_{\alpha}, Q_{\alpha}\right\rangle_{\alpha \in A}
$$

if and only if for each $\beta \in A$ there is a finite sequence $u_{\beta}$ of elements of $\beta$ such that

$$
q_{\beta}=p_{\widehat{\beta}} u_{\beta}, \quad O_{\mathscr{P}(A \cap \beta)} \Vdash Q_{\beta} \subseteq P_{\beta},
$$

and

$$
\left\langle q_{\alpha}, Q_{\alpha}\right\rangle_{\alpha \in A \cap \beta} \text { IF "every element of } u_{\beta} \text { lies in } P_{\beta} \text { ". }
$$

Two details deserve mention. First, in defining $\mathscr{P}(A)$ we must restrict our terms $P_{\alpha}$ to lie in some sufficiently large predetermined set. Otherwise, $\mathcal{P}(A)$ would be a proper class. This set of terms may be chosen so that the cardinality of $\mathscr{P}(A)$ remains as small as possible:

$$
|\mathcal{P}(A)|=\sup \left\{2^{\alpha} \mid \alpha \in A\right\} .
$$

Secondly, a passage to equivalence classes is necessary to make $<$ a true partial ordering. For more details and proofs of (I)-(V), see $\$ \S 2$ and 3 of [1].

In what follows, it will be more convenient to work with a slightly different partial order, $\mathscr{P}^{\prime}(A)$. The advantage of $\mathscr{P}^{\prime}(A)$ is that it is homogeneous in a very strong way (Lemma 2.2). A consequence of Lemma 2.1 is that forcing with $\mathscr{P}^{\prime}(A)$ is equivalent to forcing with $\mathscr{P}(A)$ in the sense that a generic object on one is definable from a generic object on the other. They produce the same generic extensions, and terms over one partial order are easily transformed into terms over the other. In particular, properties (I)-(V) hold for $\mathcal{P}^{\prime}(A)$ as well as for $\mathcal{P}(A)$.

The set $\mathscr{P}^{\prime}(A)$ is defined by induction on $\bar{A}$, similar to the definition of $\mathcal{P}(A)$. A typical condition is of the form $\left\langle s_{\alpha}, S_{\alpha}\right\rangle_{\alpha \in A}$ where $s_{\alpha}$ is a finite subset of $\alpha, S_{\alpha}$ is a term such that $O_{\mathcal{P}^{\prime}(A \cap \alpha)} \Vdash S_{\alpha} \in \tilde{\mu}_{\alpha}$, and only finitely many of the $s_{\alpha}$ 's are nonempty. The difference between such a condition and an element of $\mathscr{P}(A)$ is that $s_{\alpha}$ is a set rather than a sequence, and it is not required that every element of $s_{\alpha}$ be less than the elements of $S_{\alpha}$.

Notice that our use of $\tilde{\mu}_{\alpha}$ requires the inductive assumption that $\mathscr{P}^{\prime}(A \cap \alpha)$ is equivalent ot $\mathscr{P}(A \cap \alpha)$. Thus, the inductive proof of Lemma 2.1 should be regarded as proceeding simultaneously with the present induction. 
The ordering on $\mathscr{P}^{\prime}(A)$ is given by

if and only if for each $\beta \in A$,

$$
\left\langle s_{\alpha}, S_{\alpha}\right\rangle_{\alpha \in A} \leqslant\left\langle t_{\alpha}, T_{\alpha}\right\rangle_{\alpha \in A}
$$

$$
s_{\beta} \subseteq t_{\beta}, \quad O_{\Phi^{\prime}(A \cap \beta)} \Vdash T_{\beta} \subseteq S_{\beta},
$$

and

$$
\left\langle t_{\alpha}, T_{\alpha}\right\rangle_{\alpha \in A \cap \beta} \Vdash t_{\beta}-s_{\beta} \subseteq S_{\beta} .
$$

Just as in the case of $\mathscr{P}(A)$ we must specify in advance a set of terms from which to select the set of terms from which to select the $S_{\alpha}$, and we must pass to equivalence classes so that $\leqslant$ is really a partial ordering.

Lemma 2.1. There is a dense subset $D(A)$ of $\mathcal{P}^{\prime}(A)$ and an isomorphism $\pi_{A}$ mapping $\mathcal{P}(A)$ onto $D(A)$. Thus, if $G$ is $M$-generic over $\mathcal{P}(A)$,

$$
\left\{s \in \mathcal{P}^{\prime}(A) \mid(\exists p \in G) \pi_{A}(p) \geqslant s\right\}
$$

is $M$-generic over $\mathcal{P}^{\prime}(A)$, and $G^{\prime}$ being $M$-generic over $\mathcal{P}^{\prime}(A)$ implies $\pi_{A}^{-1}\left[G^{\prime}\right]$ is $M$-generic over $\mathscr{P}(A)$.

Proof. Let $D(A)=\left\{\left\langle s_{\alpha}, S_{\alpha}\right\rangle_{\alpha \in A} \varepsilon^{\mathcal{P}^{\prime}}(A) \mid\right.$ for all $\beta \in A, O_{\Phi^{\prime}(A \cap \beta)} \Vdash \cup s_{\beta}$ $\left.<\cap S_{p}\right\}$. The set $D(A)$ is dense in $\mathcal{P}^{\prime}(A)$, because any condition $\left\langle t_{\alpha}, T_{\alpha}\right\rangle_{\alpha \in A}$ has an extension $\left\langle t_{\alpha}, T_{\alpha}-\left[\left(\cup t_{\alpha}\right)+1\right]\right\rangle_{\alpha \in A}$ which is in $D(A)$. (We use $T_{\alpha}-\left[\left(\cup t_{\alpha}\right)+1\right]$ as an abbreviation for the term which denotes the desired set.)

We make the inductive assumption that the isomorphism $\pi_{A \cap \beta}$ between $\mathscr{P}(A \cap \beta)$ and $D(A \cap \beta)$ has been established for all $\beta \in A$. This allows us to transform a term in the language for forcing over $\mathcal{P}(A \cap \beta)$ into a term in the language for $\mathcal{P}^{\prime}(A \cap \beta)$, and vice versa.

The isomorphsim $\pi_{A}: \mathscr{P}(A) \rightarrow D(A)$ is defined by

$$
\pi_{A}\left(\left\langle p_{\alpha}, P_{\alpha}\right\rangle_{\alpha \in A}\right)=\left\langle s_{\alpha}, S_{\alpha}\right\rangle_{\alpha \in A}
$$

where $s_{\alpha}$ is the set of ordinals appearing in the sequence $p_{\alpha}$, and $S_{\alpha}$ is the term (over $\mathscr{P}^{\prime}(A \cap \alpha)$ ) which is the transformation of the term $\boldsymbol{P}_{\alpha}$. One can define an inverse $\rho_{A}: D(A) \rightarrow \mathscr{P}(A)$ similarly. The action of $\rho_{A}$ is to restore the ordering of the sequence and transform terms in the opposite direction. The reader may verify that $\pi_{A}$ and $\rho_{A}$ preserve order and that the composite maps $\pi_{A} \circ \rho_{A}$ and $\rho_{A} \circ \pi_{A}$ are the identities on equivalence classes of conditions.

For the remainder of this paper we shall work with $\mathcal{P}^{\prime}(A)$ rather than $\mathscr{P}(A)$. One of the consequences of Lemma 2.1 is that $\mathscr{P}^{\prime}(A)$ is isomorphic in $M$ to $\mathscr{P}^{\prime}(A \cap \gamma) * \mathscr{P}^{\prime}(A-\gamma)$. Thus, $\mathscr{P}^{\prime}(A \cap \gamma)$ is embedded in $\mathscr{P}^{\prime}(A)$ and we shall identify $\mathscr{P}^{\prime}(A \cap \gamma)$ with its image. If $s=\left\langle s_{\alpha}, S_{\alpha}\right\rangle_{\alpha \in A} \in \mathscr{P}^{\prime}(A)$, then $s \mid \gamma$ is the image under this embedding of $\left\langle s_{\alpha}, S_{\alpha}\right\rangle_{\alpha \in A \cap \gamma}$. If $G$ is a generic subset of $\mathcal{P}^{\prime}(A)$ then 


$$
G_{\gamma}=G \cap \mathscr{P}^{\prime}(A \cap \gamma)=\{s|\gamma| s \in G\}
$$

is a generic subset of $\mathscr{P}^{\prime}(A \cap \gamma)$. If $F$ is a function with domain $\mathscr{P}^{\prime}(A)$, then $F \mid \gamma$ will denote the restriction $\left.F\right|^{\prime}(A \cap \gamma)$.

LeMmA 2.2. If $\gamma\left\langle\kappa, s=\left\langle s_{\alpha}, S_{\alpha}\right\rangle_{\alpha \in A}\right.$ and $t=\left\langle t_{\alpha}, T_{\alpha}\right\rangle_{\alpha \in A}$ are elements of $\mathcal{P}^{\prime}(A)$, and $t$ extends $s \mid \gamma$, then there is an automorphism $F$ of $\mathcal{P}^{\prime}(A)$ such that $F \mid \gamma=\mathrm{id}$ and $F(s)$ and $t$ are compatible.

Proof. There are extensions $s^{\prime}$ and $t^{\prime}$ of $s$ and $t$, respectively, which satisfy $s^{\prime}\left|\gamma=t^{\prime}\right| \gamma$ and, for all $\alpha \in A-\gamma,\left|s_{\alpha}^{\prime}\right|=\left|t_{\alpha}^{\prime}\right|$. For each $\alpha \in A-\gamma$, let $f_{\alpha} \in M$ be a permutation of $\alpha$ which is the identity on $\alpha-\left(s_{\alpha}^{\prime} \cup t_{\alpha}^{\prime}\right)$ and satisfies $f_{\alpha}^{\prime \prime} s_{\alpha}^{\prime}=t_{\alpha}^{\prime}$. For $\alpha \in A \cap \gamma$, take $f_{\alpha}$ to be the identity on $\alpha$. Now define an automorphism $F$ of $\mathcal{P}^{\prime}(A)$ by

$$
F\left(\left\langle u_{\alpha}, U_{\alpha}\right\rangle_{\alpha \in A}\right)=\left\langle f_{\alpha}^{\prime \prime} u_{\alpha}, f_{\alpha}^{\prime \prime} U_{\alpha}\right\rangle_{\alpha \in A} .
$$

(As usual, $f_{\alpha}^{\prime \prime} U_{\alpha}$ is an abbreviation for the appropriate term.) The verification that $F$ is an automorphism of $\mathcal{P}^{\prime}(A)$ and $F \mid \gamma=\mathrm{id}$ is routine and omitted. The compatibility of $f(s)$ and $t$ is affirmed by the condition $\left\langle t_{\alpha}^{\prime}, T_{\alpha}^{\prime} \cap\right.$ $\left.f^{\prime \prime} S_{\alpha}^{\prime}\right\rangle_{\alpha \in A}$.

Let us move now to the main topic of this section. We begin with a model of $\mathrm{ZFC}+$ "there is a cardinal $\kappa$ which has normal measure $\nu$ concentrating on measurables". Let $A$ be the set of measurable cardinals below $\kappa$, and for $\alpha \in A$ let $\mu_{\alpha}$ be a normal measure on $\alpha$ which does not concentrate on measurables. Then the filter $\mu$, defined by

$$
x \in \mu \equiv x \subseteq \kappa \text { and }\left\{\gamma \in A \mid x \cap \gamma \in \mu_{\gamma}\right\} \in \nu,
$$

is a normal measure on $\kappa$ not containing $A$. Let $M$ be the inner model $L\left[\left\langle\mu_{\alpha} \mid \alpha \in A\right\rangle, \mu, \nu\right]$. By results of Mitchell [3], $M$ is a model for ZFC + $\mathrm{GCH}$, and each $\alpha \in A$ has exactly one normal measure in $M$ (namely $\mu_{\alpha} \cap M$, which will be abbreviated to $\mu_{\alpha}$ ); $\kappa$ has exactly two normal measures, $\mu$ and $\nu$; and there are no other measurable cardinals. We take $M$ to be our ground model.

As is the custom, we assume $M$ is countable, standard, and transitive. We seek an extension $N$ of $M$ which will satisfy Theorem 2.3. The reader may, if desired, rephrase the theorem as a relative consistency result.

THEOREM 2.3. The model $M$ has an extension $N$ such that:

(a) $N \vDash \mathrm{ZF}+(\forall \alpha<\kappa) \mathrm{DC}_{\alpha}$.

(b) $\operatorname{Card}^{N}=\operatorname{Card}^{M}$.

(c) $\alpha$ is regular in $N$ iff $\alpha$ is regular in $M$ and $\alpha \notin A$. (In particular, $\kappa$ remains inaccessible.)

(d) All elements of $A$ have cofinality $\omega$ in $N$.

(e) $\kappa$ is the only measurable cardinal in $N$ and has exactly two normal measures, $\bar{\mu}$ and $\bar{\nu}$, which satisfy $\{\alpha \in \kappa \mid \alpha$ is regular $\} \in \bar{\mu}$ and $\mu_{\omega} \subseteq \bar{\nu}$. 
Let $G$ be an $M$-generic subset of $\mathscr{P}^{\prime}(A)$. The model $M[G]$ is constructed from terms in the language for forcing with $\mathscr{P}^{\prime}(A)$. If $\tau$ is such a term, $\operatorname{den}_{G} \tau$ stands for the corresponding element of $M[G]$. For each $x \in M$, there is a canonical term $\mathbf{x}$ such that $x=\operatorname{den}_{G} \mathbf{x}$. Similarly, $\mathbf{G}$ is a term denoting $G$; $G=\operatorname{den}_{G} \mathbf{G}$.

If $F$ is an automorphism of $\mathscr{P}^{\prime}(A)$, then $\tau(F)$ is that term which results from replacing every occurrence of $\mathbf{G}$ in $\tau$ with a term denoting $F^{\prime \prime} G$. For a formula $\Phi$ in the appropriate language, $\Phi(F)$ has an analogous definition. It follows, then, that $P \vDash \Phi$ is equivalent to $F(p) \vDash \Phi\left(F^{-1}\right)$.

If $\gamma<\kappa$, the term $\tau$ has support $\gamma$ if for every automorphism $F$ of $\mathcal{P}^{\prime}(A)$, $F \mid \gamma=$ id implies that each condition in $\mathscr{P}^{\prime}(A)$ forces $\tau(F)=\tau$. Intuitively this means that membership facts about $\tau$ are decided by elements in $G_{\gamma}$. For $x \in M$, the term $\mathbf{x}$ is supported by zero. The model $N$ is defined by

$$
N=\bigcup_{\alpha \in \text { Ord }} N_{\alpha}
$$

where

$$
\begin{array}{r}
N_{\alpha}=\left\{x \in M[G] \mid x \subseteq \bigcup_{\beta<\alpha} N_{\beta} \text { and there exists a term } \tau \text { and an ordinal } \gamma\right. \\
\text { satisfying den } \left.\operatorname{den}_{G} \tau=x, \gamma<\kappa \text {, and } \tau \text { admits support } \gamma\right\} .
\end{array}
$$

For all $\gamma<\kappa, G_{\gamma} \in N$ and $M\left[G_{\gamma}\right] \subseteq N$.

LEMMA 2.4. If $\alpha<\kappa, f: \alpha \rightarrow M$, and $f \in M[G]$, then $f \in M\left[G_{\gamma}\right]$ for some $\gamma<\kappa$.

Proof. Case 1. range $f \subseteq \kappa$. As $\kappa$ is regular in $M[G], f$ may be coded as a subset of $\beta<\kappa$. Let $\gamma$ be the least element of $A$ which is greater than $\beta$. By property $(\mathrm{V})$ of $\mathscr{P}^{\prime}(A), M[G]=M\left[G_{\gamma}\right][H]$ where $H$ is $M\left[G_{\gamma}\right]$-generic over $\mathscr{P}^{\prime}(A-\gamma)^{M\left[G_{\gamma}\right]}$. Applying property (IV) in $M\left[G_{\gamma}\right]$ to $\mathscr{P}^{\prime}(A-\gamma)^{M\left[G_{\gamma}\right]}$, we see that $f \in M\left[G_{\gamma}\right]$.

Case 2. Otherwise. Note that $\mathscr{P}^{\prime}(A)$ satisfies the $\kappa^{+}$-antichain condition. Thus, there is a set $x \in M$, range $f \subseteq x$ and $|x|=\kappa$. Let $g: x \rightarrow \kappa$ be a one-to-one correspondence, $g \in M$. By Case $1, g \circ f \in M\left[G_{\gamma}\right]$ for some $\gamma<\kappa$ and so $f \in M\left[G_{\gamma}\right]$.

LEMMA 2.5. If $\alpha<\kappa, f: \alpha \rightarrow N$, and $f \in M[G]$, then $f \in N$.

Proof. In $M[G]$ there are functions $g: \alpha \rightarrow M$ and $h: \alpha \rightarrow \kappa$ which satisfy

"for each $\beta<\alpha, g(\beta)$ is a term such that $f(\beta)=\operatorname{den}_{G} g(\beta), h(\beta)$ supports $g(\beta)$ and range $h \subseteq \delta<\kappa$."

By Lemma 2.4, $g$ and $h$ lie in $M\left[G_{\gamma}\right]$ for some $\gamma<\kappa$. We may assume $\delta \leqslant \gamma$. Being an element of $M\left[G_{\gamma}\right], g=\operatorname{den}_{G} \tau$ for some term $\tau$ having support 
$\gamma$. Now,

$$
f=\left\{\langle\beta, x\rangle \mid \beta\left\langle\alpha \text { and }(\exists y)\left(\langle\beta, y\rangle \in \operatorname{den}_{G} \tau \text { and } x=\operatorname{den}_{G} y\right)\right\} .\right.
$$

From this description of $f$ the reader may construct a term $\sigma$ which denotes $f$. The term $\sigma$ will be supported by $\gamma$ because $\tau$ is.

LEMMA 2.6. If $x$ is a set of ordinals and $x \in N$, then $x \in M\left[G_{\gamma}\right]$ for some $\gamma<\kappa$.

Proof. Let $\mathbf{x}$ be a term denoting $x$ such tha $\gamma$ supports $\mathbf{x}$. We shall prove that all values of the formula $\alpha \in \mathrm{x}$ are decided by elements of $G_{\gamma}$ :

$$
s \Vdash \alpha \in \mathbf{x} \text { implies } s \mid \gamma \Vdash \boldsymbol{\alpha} \in \mathbf{x} \text {. }
$$

Suppose this fails - for some $s$ and $\alpha, s \Vdash \alpha \in \mathbf{x}$, but $s \mid \gamma$ has an extension $t$, $t \Vdash \alpha \notin \mathbf{x}$. The hypotheses of Lemma 2.2 are satisfied by $s$ and $t$. There is an automorphism $F$ of $\mathscr{P}^{\prime}(A)$ such that $F(s)$ and $t$ are compatible and $F \mid \gamma=$ id. Since $s \Vdash \boldsymbol{\alpha} \in \mathbf{x}$,

$$
F(s) \Vdash \alpha\left(F^{-1}\right) \in \mathbf{x}\left(F^{-1}\right) .
$$

But $\boldsymbol{\alpha}$ and $\mathbf{x}$ are supported by $\gamma$, so that

$$
F(s) \Vdash \boldsymbol{\alpha} \in \mathbf{x} \text {. }
$$

This is a contradiction to the facts that $F(s)$ is compatible with $t$ and $t \Vdash \alpha \notin \mathbf{x}$.

A similar argument proves

$$
s \Vdash \boldsymbol{\alpha} \notin \mathbf{x} \text { implies } s \mid \gamma \Vdash \boldsymbol{\alpha} \notin \mathbf{x} .
$$

Using $\mathbf{x}$, it is now possible to find a term $\tau$ in the language for forcing with $\mathscr{P}^{\prime}(A \cap \gamma)$ satisfying $x=\operatorname{den}_{G_{\gamma}} \tau$.

Proof of Theorem 2.3. (a) The proof that $N \vDash Z F$ is standard. The proof that $N \vDash(\forall \alpha<\kappa) D_{\alpha}$ follows from Lemma 2.5 and the fact that $M[G] \vDash A C$.

(b) Cardinals are preserved because $N$ is sandwiched between $M$ and $M[G]$. Property (I) of $\mathscr{P}(A)$ shows cardinals are preserved in the passage from $M$ to $M[G]$.

(c) The proof is the same as that of (b), using property (II).

(d) Fix $\alpha \in A$. By the second clause of property (II), there is in $M[G]$ a map $f: \omega \rightarrow \alpha$ which is cofinal in $\alpha$. By Lemma $2.4, f \in M\left[G_{\gamma}\right]$ for some $\gamma<\kappa$, and $M\left[G_{\gamma}\right] \subseteq N$.

(e) We define $\bar{\mu}$ and $\bar{\nu}$ to be the filters in $N$ generated by $\mu$ and $\nu$, respectively. For example,

$$
x \in \bar{\mu} \equiv \text { for some } y \in \mu, \quad y \subseteq x \subseteq \kappa .
$$

We use Lemma 2.6 and ideas of Lévy and Solovay [6] concerning mild Cohen extensions. Note that for $\gamma<\kappa,\left|\mathscr{P}^{\prime}(A \cap \gamma)\right|<\kappa$, so that $M\left[G_{\gamma}\right] \mathrm{F}^{\prime \prime} \bar{\mu} \cap$ $M\left[G_{\gamma}\right]$ is a normal measure on $\kappa$." If $x \in 2^{\kappa} \cap N$, then $x \in M\left[G_{\gamma}\right]$ for some $\gamma<\kappa$ and, consequently, either $x$ or $\kappa-x$ contains an element of $\mu$. This 
shows $N \vDash$ " $\bar{\mu}$ is an ultrafilter." The proofs for additivity and normality proceed similarly.

By part (c),

$\{\alpha \in \kappa \mid \alpha$ is regular in $N\}=\{\alpha \in \kappa \mid \alpha$ is regular in $M$ and $\alpha \notin A\}$, and the set on the right is in $\mu$.

The proof that $\bar{\nu}$ is a normal measure is identical. To see that $\mu_{\omega} \subseteq \bar{\nu}$ we note $A \in \nu$ and by part (d), $A \subseteq(\kappa)_{\omega}$. Now apply Lemma 0.1 .

We next show that if $\beta \neq \kappa$, then $\beta$ is not measurable in $N$. For the first case, assume $\beta>\kappa$. Observe that (under the correct choice for sets of terms $\left.S_{\alpha}\right),\left|\mathscr{P}^{\prime}(S)\right|^{M}=\kappa$. By an inessential modification of a well-known proof in Lévy and Solovay [6], if $\beta$ were measurable in $N$, then it would be measurable in $M$. No cardinal greater than $\kappa$ is measurable in $M$.

The remaining case is $\beta<\kappa$. We may assume $\beta \notin A$. Note that, by property (IV),

$$
2^{\beta} \cap M[G]=2^{\beta} \cap M\left[G_{\beta+1}\right]=2^{\beta} \cap N .
$$

Thus a measure on $\beta$ in $N$ is actually a measure in $M[G]$. But by property (III), $\beta$ is not measurable in $M[G]$.

It remains only to show that there are no other normal measures on $\kappa$. Let $\sigma$ be a normal measure and let $\gamma$ support $\sigma$. By the usual symmetry arguments,

$$
\sigma \cap M\left[G_{\gamma}\right] \in M\left[G_{\gamma}\right]
$$

(i.e., facts about $\sigma \cap M\left[G_{\gamma}\right]$ are determined by $G_{\gamma}$ ), and

$$
M\left[G_{\gamma}\right] \vDash \text { " } \sigma \cap M\left[G_{\gamma}\right] \text { is a normal measure on } \kappa \text { ". }
$$

By the fact that $|\mathcal{P}(A \cap \gamma)|^{M}<\kappa$, the result of Lévy and Solovay [6] already mentioned, and the choice of $M$, we must have

$$
\mu \subseteq \sigma \cap M\left[G_{\gamma}\right] \text { or } \nu \subseteq \sigma \cap M\left[G_{\gamma}\right] .
$$

Consequently,

$$
\bar{\mu}=\sigma \text { or } \bar{\nu}=\sigma .
$$

3. This section consists of an outline of the modifications to the proofs in $\S 2$ which are necessary to prove Theorem 3.1 . We begin with the ground model $M$ of $\$ 2$.

THEOREM 3.1. There is an extension $N^{\prime}$ of $M$ satisfying the following:

(a) $N^{\prime} \vDash \mathrm{ZF}+\mathrm{DC}_{\omega}$.

(b) $N^{\prime} k^{\prime \prime} \kappa_{1}=\aleph_{1}^{M}$ and $\aleph_{2}=\kappa "$.

(c) Cardinals and regular cardinals above $\kappa$ are preserved.

(d) There are exactly two normal measures on $\aleph_{2}$ in $N^{\prime}$. One extends $\mu_{\omega}$, and the other extends $\mu_{\kappa_{1}}$. 
The main difference between the proof of the present theorem and that of Theorem 2.3 is that a different partial ordering is used. The lemmas of this section are essentially adaptations of the lemmas of the previous section to the new partial ordering.

The first part of this section is devoted to a cardinal collapsing ordering $Q(\delta)$. This is followed by a discussion of the composite ordering $\mathscr{P}^{\prime}(A) * Q(\kappa)$. Next, a notion of support and the model $N^{\prime}$ are defined, and finally, it is proved that $N^{\prime}$ satisfies Theorem 3.1.

Suppose $\delta$ is regular and $\delta>\aleph_{1}$. Let $Q(\delta)$ be the collection of forcing conditions which gently collapses $\delta$ to $\aleph_{2}$. A condition is a partial function $r$ from $\aleph_{1} \times \delta$ into $\delta$ which satisfies

(1) dom $r$ is countable, and

(2) if $\langle\eta, \xi\rangle \in \operatorname{dom} r$, then $r\langle\eta, \xi\rangle\langle\xi$.

This forcing adds no new countable sets of ordinals, makes $\delta=\aleph_{2}$, and preserves cardinals and regular cardinals which are greater than $\delta$.

If $\gamma<\delta$, let

$$
Q(\gamma, \delta)=\left\{r \in Q(\delta) \mid \operatorname{dom} r \subseteq \aleph_{1} \times(\delta-\gamma)\right\} .
$$

Then there is a natural decomposition

$$
Q(\delta)=Q(\gamma) \times Q(\gamma, \delta) .
$$

If under this identification $q=\left\langle q_{0}, q_{1}\right\rangle$, then $q \mid \gamma$ is the condition

$$
q \mid \gamma=\left\langle q_{0}, 0\right\rangle \text {. }
$$

As in the case of $\mathscr{P}^{\prime}(A \cap \gamma)$ and $\mathscr{P}^{\prime}(A)$, we are identifying $Q(\gamma)$ with its image in $Q(\delta)$ :

$$
q \mid \gamma \in Q(\gamma) \subseteq Q(\delta) .
$$

We shall need a well-known result about the homogeneity of the orderings $Q(\delta)$ and $Q(\gamma, \delta)$.

LEMMA 3.2. (a) $Q(\gamma, \delta)$ is homogeneous. That is, for any two conditions $r$ and $s$, there is an automorphism $F$ of $Q(\gamma, \delta)$ such that $F(r)$ is compatible with $s$.

(b) If $\gamma<\delta$ and $r$ and $s$ are elements of $Q(\delta)$ such that $r \mid \gamma$ and $s \mid \gamma$ are compatible, then there is an automorphism $F$ of $Q(\delta)$ such that $F \mid Q(\gamma)$ is the identity and $F(r)$ is compatible with $s$.

Proof. (a) Let $r, s \in Q(\gamma, \delta)$ be given. Let $\pi$ be a permutation of $\aleph_{1} \times(\delta$ $-\gamma)$ such that

(1) if $\pi\langle\eta, \xi\rangle=\left\langle\eta^{\prime}, \xi^{\prime}\right\rangle$, then $\xi=\xi^{\prime}$, and

(2) $\left(\pi^{\prime \prime} \operatorname{dom} r\right) \cap \operatorname{dom} s=\varnothing$.

The permutation $\pi$ may be constructed by "piecing together" a suitable sequence $\left\langle\pi_{\xi} \mid \gamma \leqslant \xi<\delta\right\rangle$ of permutations of $\aleph_{1}$. For $t \in Q(\gamma, \delta)$ define $F(t)$ to be that element of $Q(\gamma, \delta)$ which satisfies

$$
\operatorname{dom} F(t)=\pi^{\prime \prime} \operatorname{dom} t \text { and } F(t)(\pi\langle\eta, \xi\rangle)=t\langle\eta, \xi\rangle .
$$


The reader may verify the fact that $F$ is an automorphism of $Q(\gamma, \delta)$. The conditions $F(r)$ and $s$ are compatible because their domains are disjoint.

(b) We use the representation $Q(\delta)=Q(\gamma) \times Q(\gamma, \delta)$. Then $r=\left\langle r_{0}, r_{1}\right\rangle$ and $s=\left\langle s_{0}, s_{1}\right\rangle$. Take $F=\operatorname{id}_{Q(\gamma)} \times F_{1}$, where $F_{1}$ is an automorphism of $Q(\gamma, \delta)$ which makes $F\left(r_{1}\right)$ compatible with $s_{1}$.

Let us now define the partial ordering required for the proof of Theorem 3.1. We work inside the model $M$, where there is a cardinal $\kappa$ admitting a measure which concentrates on a set $A$ of measurable cardinals. The ordering $\mathcal{P}^{\prime}(A)$ was defined and used in $\S 2$. For the present purpose we shall need the composition $\mathcal{P}^{\prime}(A) * Q(\kappa)$, where by $Q(\kappa)$ is meant a term in the language appropriate for $\mathcal{P}^{\prime}(A)$ which always denotes $Q(\kappa)$ in $M[G]$. In $M$, there is a set $R$ of terms such that $r \in R$ implies that $O_{\Phi^{\prime}(A)} \Vdash r \in Q(\kappa)$, and if $M[G] \vDash$ $x \in Q(\kappa)$, then $x=\operatorname{den}_{G} r$ for some $r \in R$. We do not specify $R$ further, except to say that it is assumed $R$ is sufficiently large; all the terms needed in the proofs of this section lie in $R$. We think of elements of $\mathcal{P}^{\prime}(A) * Q(\kappa)$ as pairs $\langle p, r\rangle \in \mathscr{P}^{\prime}(A) \times R$.

(Notation. We shall need to distinguish between forcing with the partial ordering $\mathscr{P}^{\prime}(A)$ and forcing with $\mathscr{P}^{\prime}(A) * Q(\kappa)$. To avoid cumbersome subscripts, we use $\leqslant$ and $\Vdash$ for the former, and $\leqslant *$ and $\Vdash^{*}$ for the latter.)

Recall that, for $\gamma<\kappa, \mathcal{P}^{\prime}(A \cap(\gamma+1))$ is a subordering of $\mathscr{P}^{\prime}(A)$. Similarly, the ordering $\mathscr{P}^{\prime}(A \cap(\gamma+1)) * Q(\gamma)$ can be considered to be a subordering of $\mathscr{P}^{\prime}(A) * Q(\kappa)$. This is done by identifying the pair $\langle p, r\rangle \in \mathscr{P}^{\prime}(A$ $\cap(\gamma+1)) * Q(\gamma)$ with the pair $\left\langle p, r^{\prime}\right\rangle$, where $r^{\prime}$ is the transformation of $r$ from a term in the language for forcing with $\mathscr{P}^{\prime}(A \cap(\gamma+1))$ into a term with support $\gamma+1$ in the language for $\mathscr{P}^{\prime}(A)$. The reason for writing $A \cap(\gamma+1)$ rather than $A \cap \gamma$ is that all countable subsets of $\gamma$ which are in $M[G]$ are actually in $M\left[G_{\gamma+1}\right]$ (property (IV) of $\mathscr{P}^{\prime}(A)$ ). Thus the version of $Q(\gamma)$ determined by forcing with $\mathcal{P}^{\prime}(A \cap(\gamma+1))$ is the same as the one obtained by forcing with $\mathcal{P}^{\prime}(A)$.

The restriction of a condition $q$ to an ordinal $\gamma$ is analogous to the notion of restriction used in $\$ 2$. If $q=\langle p, r\rangle \in \mathscr{P}^{\prime}(A) * Q(\kappa)$ and $\gamma<\kappa$, then

$$
q \mid \gamma=\langle p|(\gamma+1), r| \gamma\rangle,
$$

where $r \mid \gamma$ is an abbreviation for the appropriate term. In the event that $r \mid \gamma$ (as a term for forcing with $\left.\mathcal{P}^{\prime}(A)\right)$ has support $\gamma+1, q \mid \gamma$ can be considered as a condition which is an element of the subordering $\mathscr{P}^{\prime}(A \cap(\gamma+1)) * Q(\gamma)$. We shall not distinguish between $r \mid \gamma$ as a term over $\mathscr{P}^{\prime}(A \cap(\gamma+1))$ and $r \mid \gamma$ as a term over $\mathscr{P}^{\prime}(A)$ which is supported by $\gamma+1$.

It is not in general true that $q \mid \gamma$ is a condition, for $r \mid \gamma$ need not have support $\gamma+1$. Fortunately, for fixed $\gamma$, the set of conditions $q=\langle p, r\rangle$ such that $r \mid \gamma$ does have support $\gamma+1$ in a dense subset of $\mathcal{P}^{\prime}(A) * Q(\kappa)$. That is the content of Lemma 3.3. 
LEMMA 3.3. For every condition $q=\langle p, r\rangle \in \mathscr{P}^{\prime}(A) * Q(\kappa)$ and every ordinal $\gamma<\kappa$, there is a condition $q^{\prime}=\left\langle p^{\prime}, r^{\prime}\right\rangle$ extending $q$ such that $r^{\prime} \mid \gamma$ has support $\gamma+1$.

Proof. Let $q=\langle p, r\rangle$ and $\gamma<\kappa$ be given. By definition of $\mathscr{P}^{\prime}(A) * Q(\kappa)$,

$$
p \Vdash r \in Q(\kappa) \text { and } p \Vdash r \mid \gamma \in Q(\gamma) \text {. }
$$

When forcing with $\mathscr{P}^{\prime}(A)$,

$$
2^{\gamma} \cap M[G]=2^{\gamma} \cap M\left[G_{\gamma+1}\right] .
$$

Consequently, there is in $M$ a set $S$ of terms, all having support $\gamma+1$, such that $M[G] \vDash x \in Q(\gamma)$ implies $x=\operatorname{den}_{G} s$ for some $s \in S$.

There is an extension $p^{\prime}$ of $p$ and a term $s \in S$ such that $p^{\prime} \Vdash r \mid \gamma=s$. Let $r^{\prime}$ be a term which denotes the result of replacing $r \mid \gamma$ with $s$; that is, for $t \in \mathcal{P}^{\prime}(A), t \Vdash r^{\prime}\langle\boldsymbol{\eta}, \boldsymbol{\xi}\rangle=\boldsymbol{\beta}$ if and only if

$$
\xi<\boldsymbol{\gamma} \text { and } \boldsymbol{t} \Vdash \boldsymbol{s}\langle\boldsymbol{\eta}, \boldsymbol{\xi}\rangle=\boldsymbol{\beta},
$$

or

$$
\xi \geqslant \gamma \text { and } t \Vdash r\langle\boldsymbol{\eta}, \boldsymbol{\xi}\rangle=\boldsymbol{\beta} .
$$

It can be verified that $q^{\prime}=\left\langle p^{\prime}, r^{\prime}\right\rangle$ extends $q=\langle p, r\rangle$. The term $r^{\prime} \mid \gamma$ has support $\gamma+1$ because $O_{\Phi^{\prime}(A)} \Vdash r^{\prime} \mid \gamma=s$ and $s$ has support $\gamma+1$.

Next, we show that $\mathscr{P}^{\prime}(A) * Q(\kappa)$ has enough automorphisms.

Lemma 3.4. Assume $\gamma<\kappa, q_{0}, q_{1} \in \mathcal{P}^{\prime}(A) * Q(\kappa), q_{0} \mid \gamma$ is a condition, and $q_{0} \mid \gamma \leqslant{ }^{*} q_{1}$. Then there is an automorphism $F$ of $\mathcal{P}^{\prime}(A) * Q(\kappa)$ which is the identity on $\mathcal{P}^{\prime}(A \cap(\gamma+1)) * Q(\gamma)$ and which makes $F\left(q_{0}\right)$ compatible with $q_{1}$.

Proof. Represent $q_{0}$ and $q_{1}$ as $\left\langle p_{0}, r_{0}\right\rangle$ and $\left\langle p_{1}, r_{1}\right\rangle$, respectively. The hypotheses imply that $p_{0} \mid(\gamma+1) \leqslant p_{1}$. By Lemma 2.2 there is an automorphism $F_{0}$ of $\mathscr{P}^{\prime}(A)$ such that $F_{0} \mid(\gamma+1)$ is the identity and $F_{0}\left(p_{0}\right)$ is compatible with $p_{1}$. The map $F_{0}$ induces an automorphism of $\mathscr{P}^{\prime}(A) * Q(\kappa)$ as follows:

$$
\langle p, r\rangle \mapsto\left\langle F_{0} p, r\left(F_{0}^{-1}\right)\right\rangle .
$$

Applying Lemma 3.2(b) in the extension by $\mathscr{P}^{\prime}(A)$ yields a term $F_{1}$ such that

$O_{\mathscr{Q}^{\prime}(A)} \Vdash$ “ $F_{1}$ is an automorphism of $Q(\kappa) ; F_{1} \mid Q(\gamma)$ is the identity; and if $r_{0}\left(F_{0}^{-1}\right)$ and $r_{1}$ are elements of $Q(\kappa)$ such that $r_{0}\left(F_{0}^{-1}\right) \mid \gamma$ and $r_{1} \mid \gamma$ are compatible, then $F_{1}\left(r_{0}\left(F_{0}^{-1}\right)\right)$ and $r_{1}$ are compatible."

If $r$ is a term for an element of $Q(\kappa)$, then $F_{1}(r)$ will represent a term denoting the result of applying $\operatorname{den}_{G} F_{1}$ to $\operatorname{den}_{G} r$.

Using $F_{0}$ and $F_{1}$, define $F$ by

$$
F\langle p, r\rangle=\left\langle F_{0}(p), F_{1}\left(r\left(F_{0}^{-1}\right)\right)\right\rangle .
$$


It is routine to check that $F$ is an automorphism of $\mathscr{P}^{\prime}(A) * Q(\kappa)$ which is the identity on $\mathcal{P}^{\prime}(A \cap(\gamma+1)) * Q(\gamma)$.

To prove that $F\left(q_{0}\right)$ and $q_{1}$ are compatible we must produce a condition which dominates both. First, note that

$$
O_{\Phi^{\prime}(A)} \Vdash r_{0}\left(F_{0}^{-1}\right)\left|\gamma=\left(r_{0} \mid \gamma\right)\left(F_{0}^{-1}\right)=r_{0}\right| \gamma .
$$

The first equality holds by the definition of restriction; the second is true because $r_{0} \mid \gamma$ is assumed to have support $\gamma+1$. Next, since $q_{0} \mid \gamma<{ }^{*} q_{1}$, we have that

$$
p_{1} \Vdash r_{0}\left|\gamma \leqslant r_{1}\right| \gamma
$$

It follows from (1) and (2) that

$$
p_{1} \Vdash \text { “ } r_{0}\left(F_{0}^{-1}\right) \mid \gamma \text { and } r_{1} \mid \gamma \text { are compatible." }
$$

By the choice of $F_{1}$,

$$
p_{1} \Vdash \text { “ } F_{1}\left(r_{0}\left(F_{0}^{-1}\right)\right) \text { and } r_{1} \text { are compatible." }
$$

It is now easy to describe a condition $q^{\prime}=\left\langle p^{\prime}, r^{\prime}\right\rangle$ which extends both $F\left(q_{0}\right)$ and $q_{1}$. The conditions $F_{0}\left(p_{0}\right)$ and $p_{1}$ are compatible; let $p^{\prime \prime}$ be a common extension. From (3),

$$
p^{\prime \prime} \Vdash \exists x\left[x \in Q(\kappa) \text { and } r_{1} \leqslant x \text { and } F_{1}\left(r_{0}\left(F_{0}^{-1}\right)\right)<x\right] .
$$

Consequently there is an extension $p^{\prime}$ of $p^{\prime \prime}$ and a term $r^{\prime}$ such that

$$
p^{\prime} \Vdash r^{\prime} \in Q(\kappa) \text { and } r_{1}<r^{\prime} \text { and } F_{1}\left(r_{0}\left(F_{0}^{-1}\right)\right)<r^{\prime} \text {. }
$$

This statement combines easily with the facts $p_{1}<p^{\prime \prime}<p^{\prime}$ and $F_{0}\left(p_{0}\right)<p^{\prime \prime}$ to prove

$$
F\left(q_{0}\right) \leqslant *\left\langle p^{\prime}, r^{\prime}\right\rangle \text { and } q_{1} \leqslant^{*}\left\langle p^{\prime}, r^{\prime}\right\rangle \text {. }
$$

Therefore $F\left(q_{0}\right)$ and $q_{1}$ are compatible and the proof of Lemma 3.4 is complete.

We can now begin to define the desired model $N^{\prime}$. Take $H$ to be an $M$-generic subset of $\mathscr{P}^{\prime}(A) * Q(\kappa)$. It is a consequence of Lemma 3.3 that

$$
H_{\gamma}=H \cap\left[\mathscr{P}^{\prime}(A \cap(\gamma+1)) * Q(\gamma)\right]
$$

is $M$-generic over $\mathcal{P}^{\prime}(A \cap(\gamma+1)) * Q(\gamma)$. From $H$ we may obtain generic subsets of $\mathcal{P}^{\prime}(A)$ and $\mathscr{P}^{\prime}(A \cap \gamma)$ which will be denoted $G$ and $G_{\gamma}$, as in $\$ 2$.

Let $\tau$ be a term in the language for forcing with $\mathscr{P}^{\prime}(A) * Q(\kappa)$. If $\gamma<\kappa$, the term $\tau$ is said to have support $\gamma$ if the relation $O_{\Phi^{\prime}(A) *}{ }_{(k)} \mathbb{1}^{*} \tau(F)=\tau$ holds whenever $F$ is an automorphism of $\mathcal{P}^{\prime}(A) * Q(\kappa)$ which is the identity on $\mathscr{P}^{\prime}(A \cap(\gamma+1)) * Q(\gamma)$.

The model $N^{\prime}$ is the inner model of $M[H]$ which consists of all those elements which hereditarily are realizations of terms admitting bounded 
support. The construction of $N^{\prime}$ parallels that of $N$ in \$2. Apparently, $M\left[G_{\gamma+1}\right] \subseteq M\left[H_{\gamma}\right] \subseteq N^{\prime}$ for $\gamma<\kappa$.

LemMa 3.5. If $f: \omega \rightarrow N^{\prime}$ and $f \in M[H]$, then $f \in N^{\prime}$.

Proof. Case 1. range $f \subseteq M$. As forcing over $Q(\kappa)$ adds no functions from $\omega$ into $M, f \in M[G]$. By Lemma 2.4, for some $\gamma<\kappa, f \in M\left[G_{\gamma}\right] \subseteq N^{\prime}$.

Case 2. Otherwise. This follows from Case 1 in the same way that Lemma 2.5 followed from Lemma 2.4.

LEMMA 3.6. If $x$ is a set of ordinals and $x \in N^{\prime}$, then $x \in M\left[H_{\gamma}\right]$ for some $\gamma<\kappa$.

Proof. Identical to the proof of Lemma 2.6, with appeals to Lemmas 3.3 and 3.4.

Proof OF THEOREM 3.1. (a) Exactly the same as the proof of Theorem 2.3(a), using Lemma 3.5.

(b) No new subsets of $\omega$ are added, so $\aleph_{1}=\aleph_{1}^{M}$. For $\gamma<\kappa$, the set $H_{\gamma+1} \in N^{\prime}$ contains information enough to insure $|\gamma|^{N^{\prime}}<\aleph_{1}$, so $\kappa \leqslant \aleph_{2}^{N^{\prime}}$. Equality is seen to hold by observing that $\kappa$ is a cardinal in $M[H]$.

(c) By antichain arguments, forcing with either $\mathscr{P}^{\prime}(A)$ or $Q(\kappa)$ preserves cardinals greater than or equal to $\kappa$. Thus we have that $M$ and $M[H]$ share the same cardinals (regular cardinals) above $\kappa$, and $M \subseteq N^{\prime} \subseteq M[H]$.

(d) The proofs that $\bar{\mu}$ and $\bar{\nu}$ are the only normal ultrafilters on $\kappa$ and $\mu_{\omega} \subseteq \bar{\nu}$ are identical to their counterparts in $\$ 2$. The main tool in those proofs was Lemma 2.6 which corresponds in the present context to Lemma 3.6. It remains only to show $\mu_{\kappa_{1}} \subseteq \bar{\mu}$. Being a normal measure in $M, \mu$ concentrates on the set $C$ of regular cardinals, and $A \notin \mu$. Lemma 3.7 will show that every element of $C-A$ has in $N^{\prime}$ cofinality $\aleph_{1}$. The result then follows from Lemma 0.1.

Lemma 3.7. If $\alpha \in C-A$, then $\operatorname{cf}^{N^{\prime}}(\alpha)=\aleph_{1}$.

Proof. Being regular in $M$ and not an element of $A, \alpha$ is regular in $M[G]$. Forcing with $Q(\kappa)$ adds no new countable sets of ordinals, so $\mathrm{cf}^{M[H]}(\alpha)>\omega$. As $|\alpha|^{N^{\prime}}=\aleph_{1}$, we are forced to conclude $\operatorname{cf}^{N^{\prime}}(\alpha)=\aleph_{1}$.

The proof of Theorem 3.1 is now complete.

\section{BiBLIOGRAPHY}

1. M. Magidor, How large is the first strongly compact cardinal?, Ann. Math. Logic 10 (1976), 33-57.

2. D. A. Martin and J. B. Paris, AD $\vdash \boldsymbol{N}_{2}$ has exactly two normal measures (manuscript).

3. W. Mitchell, Sets constructible from sequences of ultrafilters, J. Symbolic Logic 39 (1974), 57-66.

4. K. Prikry, Changing measurable cardinals into accessible cardinals, Dissertationes Math. 68 (1970), 5-52. 
5. J. Schoenfield, Unramified forcing, Proc. Sympos. Pure Math., vol. 13, Amer. Math. Soc., Providence, R. I., 1976, pp. 357-382.

6. A. Lévy and R. M. Solovay, Measurable cardinals and the continum hypothesis, Israel J. Math. 5 (1967), 234-248.

Department of Mathematics, Universtty of California, los Angeles, California 90024 Department of Mathematics, Massachusetts institute of Technology, Cambridge, MASSACHUSETTS 02139

Current address (E. L. Bull): Department of Mathematics, Pomona College, Claremont, California 91711

Current address (E. M. Kleinberg): Department of Mathematics, State University of New York at Buffalo, Buffalo, New York 14214 